\title{
LRS1: UN ROBOT SOCIAL DE BAJO COSTO PARA LA ASIGNATURA "PROGRAMACIÓN 1"
}

\section{LRS1: A LOW COST SOCIAL ROBOT FOR THE COURSE "PROGRAMMING 1"}

\author{
MSc. Jesús Pérez*, Br. José Castro* \\ * Laboratorio de Sistemas Discretos, Automatización e Integración. \\ Universidad de Los Andes, Mérida, Venezuela. \\ E-mail: \{jesuspangulo, castroj\}@ula.ve.
}

\begin{abstract}
Resumen: Los robots sociales han generado efectos positivos en el contexto educativo a través de diferentes roles: tutor, compañero y aprendiz. Los estudiantes de la asignatura "Programación 1" se han mostrado desmotivados en los últimos semestres. En aras de motivar el aprendizaje de los estudiantes de "Programación 1" se propone la incorporación de un robot social dentro de la dinámica de las clases. La adquisición de robots sociales comerciales es muy costosa, por lo tanto, se construye un robot social minimalista con tres habilidades principales: hablar, cambiar la expresión facial (triste, normal y feliz) y mover el cuello (arriba, abajo, izquierda, derecha y centro). El robot social construido (denominado LRS1) fue incorporado como tutor en una actividad denominada "dictado" que pertenece al normal desarrollo de la asignatura, cuyos resultados obtenidos favorecen la incorporación de un robot social de bajo costo para motivar el aprendizaje en la asignatura.
\end{abstract}

Palabras clave: robot social, curso de programación, motivación con robótica.

\begin{abstract}
Social robots have generated positive effects in the educational context through different roles: tutor, partner and learner. The students of the "Programming 1" course have shown demotivated in the last semesters. In order to motivate the students' learning of the "Programming 1" course, the incorporation of a social robot within the dynamics of the classes is proposed. The acquisition of commercial social robots is very expensive; therefore, a minimalist social robot is built with three main skills: talk, change the facial expression (sad, normal and happy) and move the neck (up, down, left, right and center). The social robot built (named LRS1) was incorporated as a tutor in an activity called "dictation" that belongs to the normal course development, whose obtained results favor the incorporation of a low cost social robot to motivate the course learning.
\end{abstract}

Keywords: social robot, programming course, motivation with robotics.

\section{INTRODUCCIÓN}

La robótica social aparece cada vez más en diferentes contextos, tales como asistencia a personas con requerimientos especiales (Pantecouteau y Passera, 2017) y educación de personas de diferentes edades (Fu y Zhang, 2015). En el contexto educativo se ha visto cómo los robots sociales han generado efectos positivos en los estudiantes, desde aumentar la tasa de compromiso en estudiantes de preescolar (Kirstein $y$ Risager, 2016), hasta aumentar la creatividad en estudiantes universitarios (Kahn et al., 2016). Los roles que han sido estudiados en la robótica social en el contexto educativo son: tutor, donde el robot actúa como profesor, es decir, enseña directamente al estudiante (Kennedy et al., 2016; Blar et al., 2014); compañero, el robot actúa como un 
acompañante del proceso de aprendizaje del estudiante y enseña implícitamente (Causo et al., 2017); y aprendiz, donde el estudiante le enseña actividades particulares al robot (Lemaignan et al., 2016).

La asignatura "Programación 1" pertenece al primer semestre de la carrera de Ingeniería de Sistemas de la Universidad de Los Andes, ubicada en Mérida-Venezuela; es de tipo obligatoria y está incluida dentro de las asignaturas del ciclo básico. $\mathrm{Su}$ objetivo principal es que los estudiantes desarrollen habilidades en el análisis, diseño y construcción de programas codificados en un lenguaje de programación de alto nivel, que permitan resolver problemas presentados en orden de complejidad creciente. $\mathrm{Su}$ contenido programático está divido en seis unidades: resumen histórico de la computadora, descripción funcional de la computadora, desarrollo de programas, lógica de programación, subprogramas, y estructuras de datos.

En los últimos semestres, se ha notado desmotivación en los estudiantes de la asignatura "Programación 1", lo cual se ve reflejado en la deserción, la tasa de inasistencias y el incumplimiento de las actividades. Entre los posibles elementos que contribuyen a esta situación se incluyen: los contenidos extensos de las unidades, la alta exigencia en las evaluaciones y la falta de adecuación al ambiente universitario. En ese sentido, en aras de motivar a los estudiantes a esforzarse más, se propone la incorporación de un robot social dentro de la dinámica de las clases.

La adquisición de los robots sociales comerciales es muy costosa, por lo tanto, en este trabajo se construye un robot social minimalista con tres habilidades principales: hablar, cambiar la expresión facial (triste, normal y feliz) y mover el cuello (arriba, abajo, izquierda, derecha y centro). El robot social construido fue nombrado LRS1 (acrónimo de Laboratorio de Sistemas Discretos, Automatización e Integración Robot Social 1) y fue incorporado como tutor en una actividad denominada "dictado" que pertenece al normal desarrollo de la asignatura, cuyos resultados obtenidos favorecen la incorporación de un robot social de bajo costo para motivar el aprendizaje en la asignatura.

\section{ESTADO DEL ARTE}

Recientemente, distintos robots sociales han sido utilizados en el contexto de la educación con diferentes aplicaciones. Entre los objetivos que se han estudiado están: en niños, enseñar un segundo idioma (Kennedy et al., 2016), comparar la capacidad de enseñanza de un robot social físico versus un agente virtual (Tozadore et al., 2017), desplegar robots como asistentes del docente en un salón de clases (Causo et al., 2017), introducir la programación mientras se juega con un robot social (Diyas et al., 2016), comparar la eficacia de un tutor humano con la de un robot (Blar et al., 2014), demostrar el éxito de los robots sociales en la educación (Kirstein y Risager, 2016), investigar el rol asignado al robot luego de una interacción prolongada (Sequeira et al., 2016), determinar la eficiencia de un sistema de aprendizaje que usa robots versus un sistema tradicional que utiliza PowerPoint (Chin et al., 2014), y presentar los métodos utilizados para el desarrollo de un robot cuyo propósito es asistir a un profesor en el salón de clases (Núñez, 2014); en jóvenes, mejorar el rendimiento con el uso de un robot educativo (Brown y Howard, 2014); y en adultos, encontrar una solución para evitar la depresión y el Alzheimer (El-Aawar, 2017).

Entre los efectos positivos que han generado los robots sociales en el contexto educativo están: ayudar significativamente en el aprendizaje del idioma francés (Kennedy et al., 2016); generar impacto en los niños por ser llamativo (Tozadore et al., 2017); motivar la interacción social entre los estudiantes y ayudar a desarrollar su creatividad (Causo et al., 2017); motivar el aprendizaje cuando actúan como profesores (Diyas et al., 2016); generar resultados similares al propio docente (Blar et al., 2014); aumentar la concentración en los niños en los juegos (Kirstein y Risager, 2016); incentivar a los niños a asignarle roles de amistad y compañerismo (Sequeira et al., 2016); aumentar la eficiencia con respecto a diapositivas de PowerPoint (Chin et al., 2014); aumentar la motivación de los estudiantes (Núñez., 2014); reducir los tiempos durante la realización de las evaluaciones (Brown $y$ Howard, 2014).

Los trabajos por realizar son muchos, entre los cuales están: extender a otras áreas de aprendizaje (Kennedy et al., 2016); incluir actividades con configuraciones más sofisticadas e interactivas en el robot (Tozadore et al., 2017), diseñar una aplicación que facilite la comunicación humanorobot (Causo et al., 2017); continuar la investigación en dirección de estudiar los robots sociales en la educación (Diyas et al., 2016); realizar experimentos similares con nuevos métodos y tecnologías (Blar et al., 2014); evaluar 
más a fondo los robots sociales en las instituciones educativas (Kirstein y Risager, 2016); continuar estudios sobre la asignación de roles a robots (Sequeira et al., 2016); desarrollar futuros experimentos que puedan aportar más conclusiones en el mejoramiento de la educación (Chin et al., 2014); examinar los efectos del compromiso social usando robots en niños con discapacidades (Brown y Howard, 2014); y hacer una aplicación pedagógica para estimular la creatividad del usuario (Núñez, 2014).

De los trabajos presentados anteriormente, se puede constatar que la aplicación más común es la utilización de robots sociales como tutores en el salón de clases y que los objetivos de las investigaciones giran alrededor de comparar la eficiencia de un tutor humano con la de un robot. En ese sentido, todavía se requieren muchas más investigaciones para extender a otras áreas de aprendizaje y crear nuevos métodos para mejorar la educación, por lo tanto, en este trabajo se diseña un robot social de bajo costo, se incorpora como tutor de la actividad "dictado" en el salón de clases de la asignatura "Programación 1" y se compara con el profesor.

\section{ROBOT SOCIAL (LRS1)}

\subsection{Especificaciones}

La construcción de LRS1 debe permitir la realización de la actividad denominada "dictado", la cual consiste en dictar programas en lenguaje natural para que los estudiantes escriban en lenguaje de programación $\mathrm{C}$ utilizando la sintaxis adecuada. En ese sentido, la principal habilidad que debe tener LRS1 es hablar.

Adicionalmente, para presentar un robot social que realmente pueda motivar a los estudiantes, se han definido dos habilidades que permitirán enriquecer la expresión del robot mientras habla, las cuales son: cambiar la expresión facial entre triste, normal y feliz; y mover el cuello hacia arriba, abajo, izquierda, derecha o centro.

El cambio de expresiones faciales se inspira en trabajos previos (Dapena et al., 2016), donde se utilizan tres métodos: changeExpression(emotion), para mostrar la expresión facial asociada a la emoción indicada; talk(), para iniciar la animación de los movimientos de la boca; y stopTalk(), para finalizar la animación de los movimientos de la boca.

\subsection{Diseño}

El diseño de LRS1 debe utilizar la menor cantidad de recursos para reducir el costo. En ese sentido, se propone la utilización de recursos que no son propios del robot, tales como: un computador para la toma de decisiones y un altavoz para la síntesis de la voz. Por lo tanto, el sistema que permite el funcionamiento del robot se divide en tres partes: robot físico, computador y altavoz, tal como se muestra en la Figura 1. La interacción entre los componentes del sistema se realiza de la siguiente manera: en el computador se alberga el componente cognitivo que controla al robot (expresiones faciales, movimientos de la boca y cuello) y envía las señales de audio al altavoz.

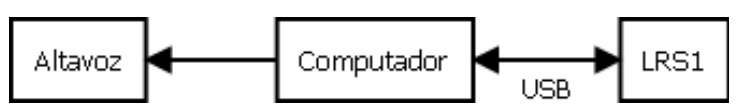

Fig. 1. Diagrama de bloques del sistema

La comunicación entre el computador y el robot físico es serial, y se realiza según el protocolo que se presenta en el diagrama de interacción de la Figura 2: primero, el computador solicita la conexión y espera respuesta; segundo, en caso de estar disponible, el robot establece la conexión y lo notifica al computador; tercero, el computador envía los comandos que controlan las expresiones del robot y las señales que se reproducen en el altavoz; y cuarto, cuando el computador termina de enviar los comandos, envía la notificación de desconexión al robot.

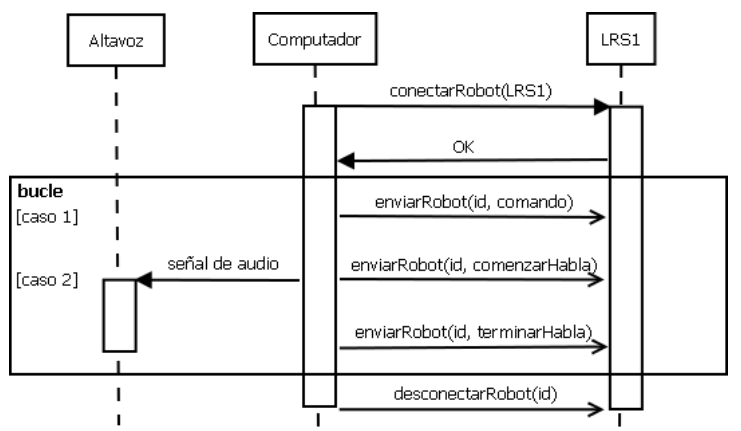

Fig. 2. Protocolo de comunicación.

El protocolo de comunicación se debe implementar tanto en el computador como en el robot. Las funciones del computador se pueden reunir en cuatro acciones: establecer la conexión con el robot, enviar los comandos para modificar las expresiones del robot, enviar las señales al altavoz y terminar la conexión con el robot. Estas cuatro acciones se incluyen en una biblioteca que ha sido denominada lasdai-ula. A continuación se presentan los prototipos de los subprogramas que 
deben ser implementados en la biblioteca lasdaiula.

- int conectarRobot(); Realiza la conexión serial entre el computador y el robot. Si la conexión es exitosa, retorna el identificador del robot.

- void hablarRobot(int, char*); Recibe dos parámetros: primero, el identificador del robot; y segundo, una cadena de caracteres. Se encarga de convertir la cadena de caracteres a voz, reproducir la voz y gestionar los movimientos de la boca del robot mientras se reproduce la voz.

- void enviarRobot(int, int); Recibe dos parámetros: primero, el identificador del robot; y segundo, un número asociado a un comando. Se encarga de enviar el comando al robot.

- void desconectarRobot(int); Recibe el identificador del robot como parámetro. Se encarga de informar al robot que ha concluido la comunicación serial.

Los comandos que se envían al robot se clasifican en dos tipos: expresiones faciales y movimientos del cuello. El nombre de los comandos se compone de dos palabras que se escriben pegadas: la primera, es un verbo en infinitivo, y la segunda, es un identificador que comienza con mayúscula. En la Tabla 1 se presentan los comandos relacionados con las expresiones faciales, los cuales pueden ser integrales (expresarNormal, expresarFeliz y expresarTriste) y particulares (cerrarIzquierdo, cerrarDerecho, abrirIzquierdo, abrirDerecho, comenzarHabla y terminarHabla). Los movimientos del cuello son independientes de las expresiones faciales $y$ tienen dos grados de libertad, de manera que los movimientos posibles son (ver Tabla 2): moverIzquierda, moverDerecha, moverArriba, moverAbajo y moverCentro.

La implementación de la biblioteca lasdai-ula se realiza en lenguaje $\mathrm{C}$, utilizando el sistema operativo Linux para su desarrollo y pruebas. Se utiliza gedit como editor de texto y gcc como compilador. Además, como sintetizador de voz se utiliza espeak, el cual debe ser instalado en el computador y se encuentra en los repositorios de Linux.

\section{Tabla 1. Comandos relacionados con las expresiones faciales.}

\begin{tabular}{|c|c|c|}
\hline $\mathbf{N}$ & Comando & Descripción \\
\hline 1 & expresarTriste & $\begin{array}{l}\text { Intensidad de LEDs baja, ojos } \\
\text { encendidos, LED del medio de la } \\
\text { primera fila de la boca encendido, } \\
\text { LEDs de los extremos del la segunda } \\
\text { fila encendidos, movimiento del } \\
\text { cuello de } 10 \text { grados hacia abajo. }\end{array}$ \\
\hline 2 & expresarNormal & $\begin{array}{l}\text { Intensidad de LEDs media, ojos } \\
\text { encendidos y primera fila de la boca } \\
\text { encendida. }\end{array}$ \\
\hline 3 & expresarFeliz & $\begin{array}{l}\text { Intensidad de LEDs alta, ojos } \\
\text { encendidos, LEDs de los extremos } \\
\text { de la primera fila de la boca } \\
\text { encendidos, LED del medio de la } \\
\text { segunda fila encendido y } \\
\text { movimiento del cuello de } 10 \text { grados } \\
\text { hacia arriba. }\end{array}$ \\
\hline 4 & cerrarIzquierdo & LED del ojo izquierdo apagado. \\
\hline 5 & cerrarDerecho & LED del ojo derecho apagado. \\
\hline 6 & abrirIzquierdo & LED del ojo izquierdo encendido. \\
\hline 7 & abrirDerecho & LED del ojo derecho encendido. \\
\hline 8 & comenzarHabla & $\begin{array}{l}\text { Comienza a simular los movimientos } \\
\text { de la boca para hablar. }\end{array}$ \\
\hline 9 & terminarHabla & $\begin{array}{l}\text { Termina de simular los movimientos } \\
\text { de la boca para hablar. }\end{array}$ \\
\hline
\end{tabular}

Tabla 2. Comandos relacionados con los movimientos del cuello

\begin{tabular}{|c|c|c|}
\hline $\mathbf{N}$ & Comando & \multicolumn{1}{|c|}{ Descripción } \\
\hline 10 & moverIzquierda & $\begin{array}{l}\text { Mueve el cuello 30 grados hacia la } \\
\text { izquierda con respecto al centro. }\end{array}$ \\
\hline 11 & moverDerecha & $\begin{array}{l}\text { Mueve el cuello 30 grados hacia la } \\
\text { derecha con respecto al centro. }\end{array}$ \\
\hline 12 & moverArriba & $\begin{array}{l}\text { Mueve el cuello 30 grados hacia } \\
\text { arriba con respecto al centro. }\end{array}$ \\
\hline 13 & moverAbajo & $\begin{array}{l}\text { Mueve el cuello 30 grados hacia } \\
\text { abajo con respecto al centro. }\end{array}$ \\
\hline 14 & moverCentro & $\begin{array}{l}\text { Mueve el cuello hacia el centro } \\
\text { (estado inicial) }\end{array}$ \\
\hline
\end{tabular}

En el controlador del robot también se debe implementar el protocolo de comunicación. Las actividades que debe realizar el controlador del robot se dividen de la siguiente manera: leer los datos que envía el computador, actualizar las expresiones integrales, actualizar los ojos, actualizar la boca y actualizar el cuello. A continuación se presentan los prototipos de los subprogramas que deben implementarse en LRS1.

- int leerSerial(); Se encarga de esperar los comandos que envía el computador. Si recibe un comando, inmediatamente lo retorna para que sea procesado. 
- void actualizarExpresion(int); Recibe como parámetro un número que indica la expresión a realizar: 1 triste, 2 normal y 3 feliz. Se encarga de ejecutar las acciones para expresar el estado indicado.

- void actualizarOjos(boolean, boolean); Recibe dos parámetros de tipo booleano: el primero, asociado al ojo izquierdo; y el segundo, relacionado con el ojo derecho. $\mathrm{Si}$ el valor es Verdadero se enciende el LED correspondiente, y si el valor es Falso se apaga.

- void actualizarBoca(boolean); Recibe un parámetro de tipo booleano que indica dos acciones posibles: Verdadero, para comenzar a simular los movimientos del habla; y Falso para terminar de simular los movimientos.

- void actualizarCuello(int); Recibe un parámetro de tipo entero que indica el movimiento del cuello: 1 izquierda, 2 derecha, 3 arriba, 4 abajo y 5 centro.

El algoritmo principal de LRS1 está basado en una estructura de decisión múltiple, donde el valor que se evalúa es el retorno de la función leerSerial, de manera que: para los comandos 1,2 y 3 se ejecuta el procedimiento actualizarExpresion; para los comandos 4, 5, 6 y 7 se ejecuta actualizarOjos; para los comandos 8 y 9 , actualizarBoca, y para los comandos $10,11,12,13$ y 14 se ejecuta actualizarCuello.

\subsection{Construcción}

En la Tabla 3 se presentan los componentes que se requieren para la construcción del robot social LRS1 según las especificaciones y diseños presentados anteriormente.

\section{Tabla 3. Componentes}

\begin{tabular}{|c|c|}
\hline Componente & Precio \\
\hline 2 LEDs verdes de $5 \mathrm{~mm}$ & $\$ 1$ \\
\hline 6 LEDs verdes rectangulares de $5 \mathrm{~mm}$ & $\$ 3$ \\
\hline 2 servomotores SG90 & $\$ 8$ \\
\hline 1 baquelita de $60 \times 40 \mathrm{~mm}$ & $\$ 2$ \\
\hline 1 baquelita de $40 \times 40 \mathrm{~mm}$ & $\$ 2$ \\
\hline 1 Arduino UNO & $\$ 24$ \\
\hline 8 cables Dupont de $150 \mathrm{~mm}$ & $\$ 1$ \\
\hline Total & $\$ 41$ \\
\hline
\end{tabular}

La conexión de los componentes se realiza según el diagrama que se presenta en la Figura 3. El
Arduino UNO es el núcleo de LRS1 y se encarga de establecer la comunicación serial con el computador, y mantener las expresiones faciales y los movimientos del cuello.
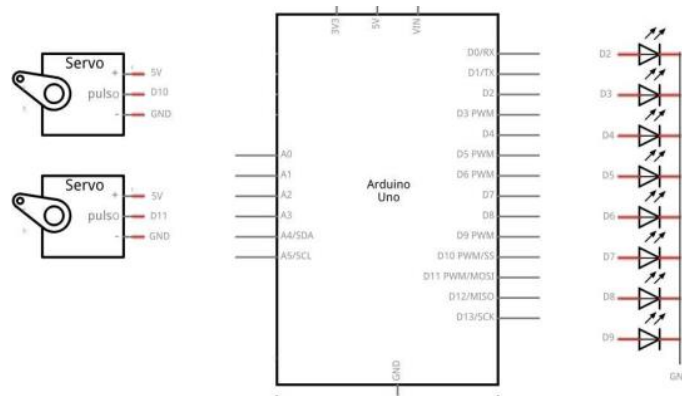

Fig. 3. Diagrama de conexiones eléctricas

El rostro se representa colocando estratégicamente los LEDs según la apariencia de un rostro humano, tal como se muestra en la Figura 4.

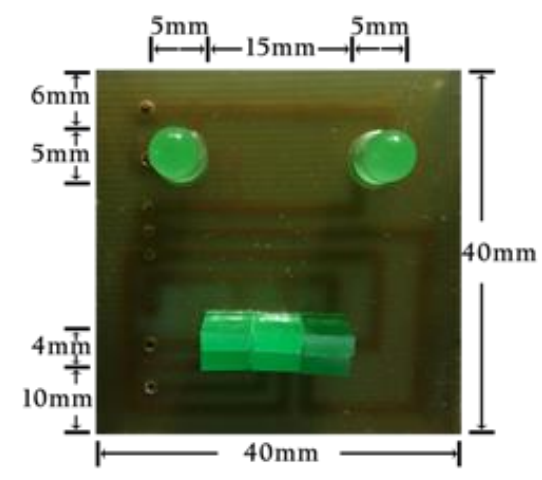

Fig. 4. Distribución del rostro

Finalmente, se conectan los demás componentes de manera que permitan los movimientos del cuello y se coloca una máscara inspirada en el robot Cody de la película "Robosapien: Rebooted", tal como se muestra en la Figura 5.

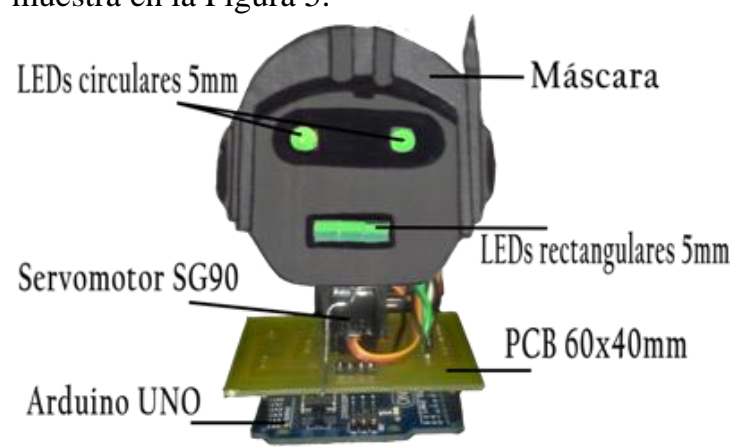

Fig. 5. Vista frontal de LRSI

En la Figura 6 se presenta la vista lateral de LRS1, donde se pueden apreciar las conexiones de los cables y las ubicaciones de los servomotores para lograr los dos grados de libertad del cuello. 


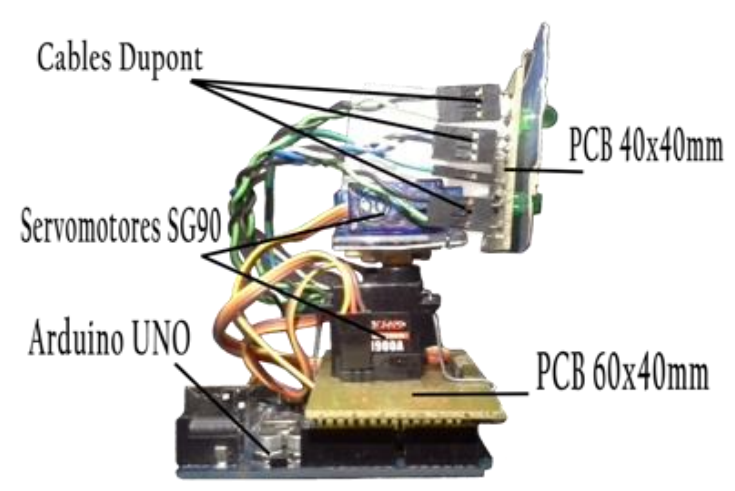

Fig. 6. Vista lateral de LRSI

La organización y conexión de los componentes generó un robot con las especificaciones físicas que se presentan en la Tabla 4.

\section{Tabla 4. Especificaciones físicas de LRS1}

\begin{tabular}{|c|c|}
\hline Atributo & Dimensión \\
\hline Alto & $105 \mathrm{~mm}$ \\
\hline Ancho & $60 \mathrm{~mm}$ \\
\hline Largo & $80 \mathrm{~mm}$ \\
\hline Peso & $80 \mathrm{~g}$ \\
\hline Grados de libertad & 2 \\
\hline
\end{tabular}

\subsection{Pruebas}

Las pruebas se realizan verificando el correcto funcionamiento de la comunicación serial entre el computador y LRS1, y comprobando la ejecución de las acciones. El programa que permite establecer la comunicación y enviar comandos al robot se presenta en la Tabla 5.

\section{Tabla 5. Programa de prueba}

\begin{tabular}{|l|l|}
\hline \multicolumn{1}{|c|}{ Instrucción } & \multicolumn{1}{c|}{ Descripción } \\
\hline \#include "lasdai-ula.h" & $\begin{array}{l}\text { Incluye la biblioteca lasdai- } \\
\text { ula. }\end{array}$ \\
\hline int main 0\{ & $\begin{array}{l}\text { Declara la función principal } \\
\text { de tipo entero }\end{array}$ \\
\hline int id = conectarRobot(LRS1); & $\begin{array}{l}\text { Realiza la conexión serial } \\
\text { con el robot LRS1 y le } \\
\text { asigna su identificador a la } \\
\text { variable id. }\end{array}$ \\
\hline enviarRobot(id,expresarFeliz); & Envía el comando al robot. \\
\hline desconectarRobot(id); & $\begin{array}{l}\text { Notifica al robot que se ha } \\
\text { concluido la comunicación. }\end{array}$ \\
\hline return $0 ;$ & $\begin{array}{l}\text { Indica que el programa se } \\
\text { ha ejecutado correctamente. }\end{array}$ \\
\hline \} & $\begin{array}{l}\text { Indica el fin de la función } \\
\text { principal. }\end{array}$ \\
\hline
\end{tabular}

Al cambiar el comando que se le envía al robot (cuarta instrucción de la Tabla 5), se puede verificar el correcto funcionamiento de todas las instrucciones. Por ejemplo, en la Figura 7 se muestran los resultados para las expresiones faciales: expresarTriste, expresarNormal y expresarFeliz.

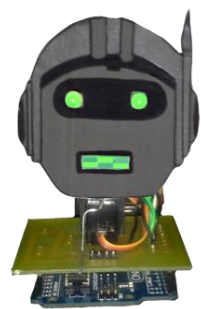

Triste

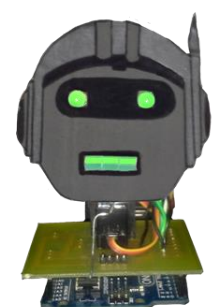

Normal

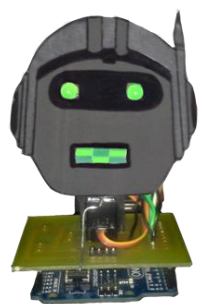

Feliz
Fig. 7. Expresiones faciales

\section{EXPERIMENTO}

El objetivo experimental es incorporar al robot social LRS1 en la actividad "dictado" para evaluar la incidencia que tiene su presencia y participación sobre los estudiantes, y además, comparar su participación como tutor con respecto al profesor de la asignatura. El estudio es realizado con un enfoque cuantitativo y un diseño de experimento puro, el cual es aquel que manipula una o más variables independientes para observar sus cambios en las variables dependientes en situaciones de control (Campbell y Stanley, 1995). En este caso, la variable independiente es el robot LRS1 como tutor y la variable dependiente es el rendimiento en la actividad "dictado". El experimento se realiza a 20 estudiantes pertenecientes a la sección 01 de la asignatura "Programación 1" del semestre A-2018 con edades comprendidas entre 17 y 20 años.

El experimento se divide en cuatro pasos principales: evaluación diagnóstica, desarrollo del experimento, aplicación de encuesta y análisis de resultados. La evaluación diagnóstica consiste en resolver 3 problemas con orden de complejidad creciente utilizando los conocimientos básicos de programación, cuyo propósito es realizar el emparejamiento para obtener dos grupos equivalentes (experimental y grupo de control) de la siguiente manera: se ordenan las calificaciones en forma descendente, se distribuyen equitativamente los estudiantes en los dos grupos según sus calificaciones (para tener precisión en la distribución de los grupos, la suma de las calificaciones de cada grupo debe ser igual), y luego, se define aleatoriamente el grupo experimental.

En el desarrollo del experimento, se aplica la actividad "dictado", donde el robot dicta 3 
ejercicios en lenguaje natural para que los estudiantes pertenecientes al grupo experimental lo codifiquen en lenguaje de programación $\mathrm{C}$ usando la biblioteca pr1-ula (biblioteca utilizada en la asignatura "Programación 1" para enseñar programación). La dinámica del dictado se realizó de la siguiente manera: primero, el robot social LRS1 se presentó ante el grupo de estudiantes mostrando cada una de sus habilidades (hablar, cambiar las expresiones faciales y mover el cuello) e indicando las instrucciones de la actividad; segundo, se efectuó el dictado de los 3 ejercicios seleccionados; y tercero, se aplicó una encuesta a los estudiantes donde se les preguntó acerca de la interacción con el robot. Por otro lado, para el grupo de control se aplica el mismo método, pero esta vez el profesor de la asignatura es quien dicta los ejercicios. Hay que resaltar que el experimento se realiza en el salón de clases el mismo día, primero al grupo de control y luego al grupo experimental.

Los resultados obtenidos se evalúan según la cantidad de líneas escritas correctamente en cada estudiante para cada uno de los 3 dictados realizados. El dictado 1 consiste en realizar un programa donde se debe incrementar el valor de una variable de tipo entero y luego mostrar por la pantalla el nuevo valor. El dictado 2 consiste en realizar un programa que asigne el doble del valor de una variable en cada posición de un arreglo de enteros, y mostrar por pantalla el valor de la segunda posición del arreglo. El dictado 3 consiste en realizar un programa que intercambie dos posiciones de un arreglo, para luego mostrar por pantalla la primera posición del arreglo. A modo de ejemplo, en la Tabla 6 se presenta el dictado 1 .

\section{Tabla 6. Dictado 1}

\begin{tabular}{|c|c|}
\hline Instrucción & Dictado 1 \\
\hline \#include "pr1-ula.h" & Incluya la biblioteca pr1-ula.h. \\
\hline int main ()\{ & $\begin{array}{l}\text { Declare la función principal de tipo } \\
\text { entero. }\end{array}$ \\
\hline int $\mathbf{a}$; & $\begin{array}{l}\text { Dentro de la función principal, } \\
\text { declare una variable de tipo entero } \\
\text { con identificador a. }\end{array}$ \\
\hline $\mathbf{a}=2$ & A la variable a, asígnele el número 2. \\
\hline $\mathbf{a + +}$ & A la variable a súmele 1 . \\
\hline imprimirEntero(a); & $\begin{array}{l}\text { Muestre el valor de la variable a en la } \\
\text { pantalla. }\end{array}$ \\
\hline return 0 ; & No olvide colorar el return 0 . \\
\hline$\}$ & $\begin{array}{l}\text { Recuerde cerrar la llave de la función } \\
\text { principal. }\end{array}$ \\
\hline
\end{tabular}

\section{RESULTADOS}

El grupo experimental seleccionado aleatoriamente fue el grupo 2. La evaluación de cada dictado consiste en dividir la nota máxima (20 puntos) entre el número de líneas de cada ejercicio, con el propósito de tener mayor precisión en el análisis de los resultados. La calificación final se calcula con el promedio de las tres notas obtenidas en los dictados.

En la Figura 8 se presenta la distribución de frecuencias del dictado 1 (el eje horizontal representa cada línea de código del dictado y el eje vertical el número de estudiantes que respondieron correctamente), donde se observa en general un mejor desempeño en el grupo de control. Particularmente, en las instrucciones 5 y 6 ningún estudiante del grupo experimental acertó el dictado.

\section{Fig. 8. Frecuencias del dictado 1}

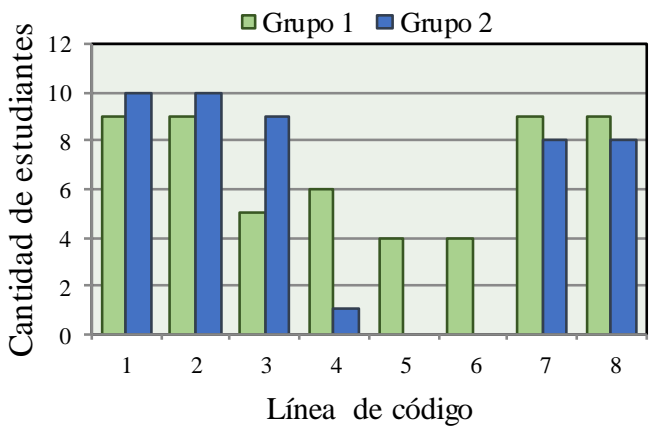

En la Figura 9 se presenta la distribución de frecuencias del dictado 2, donde se observa en general un mejor desempeño en el grupo experimental. Particularmente, en las instrucciones $1,2,3,5,6$ y 8 , los resultados fueron iguales, y en las instrucciones $4,7,9$ y 10 los resultados del grupo experimental fueron mejores.

\section{Fig. 9. Frecuencias del dictado 2}

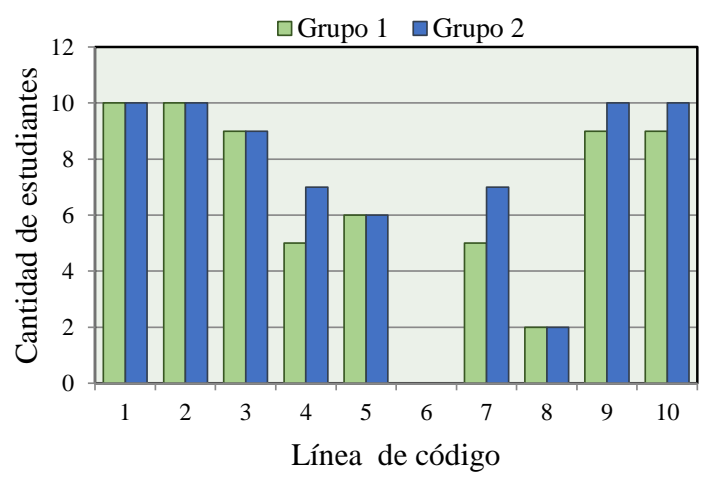


En la Figura 10 se presenta la distribución de frecuencias del dictado 3 , donde se observa mejor desempeño en el grupo experimental. En las instrucciones $1,3,4,6,9$ y 10 los resultados fueron iguales, y en las instrucciones 2, 5, 7 y 8 los resultados del grupo experimental fueron mejores.

\section{Fig. 10. Frecuencias del dictado 3}

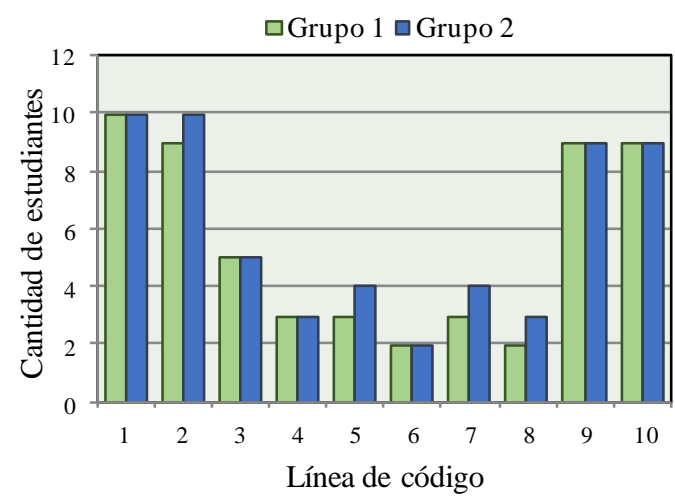

Los parámetros estadísticos usados para analizar las calificaciones finales se presentan en la Tabla 7. En el grupo de control, la calificación más alta y baja fueron 19 y 8 respectivamente; la moda y la mediana fueron 13 , y el promedio fue 12,4 con una desviación de 3,34 puntos. En el grupo experimental, la calificación más alta y baja fueron 15 y 7 respectivamente; la moda fue 14 , la mediana fue 12,5 y el promedio 12,1 con una desviación de 2,42 puntos. En general, los resultados favorecen al grupo de control porque aunque obtuvo mayor dispersión, su promedio es mayor.

\section{Tabla 7. Estadística de las calificaciones.}

\begin{tabular}{|l|c|c|}
\hline \multicolumn{1}{|c|}{ Variable } & \multicolumn{2}{c|}{ Resultados } \\
\hline Moda & Grupo 1 & Grupo 2 \\
\hline Mediana & 13 & 14 \\
\hline Media & 13 & 12,5 \\
\hline Desviación estándar & 12,4 & 12,1 \\
\hline Puntuación más alta & 3,34 & 2,42 \\
\hline Puntuación más baja & 19 & 15 \\
\hline
\end{tabular}

Los resultados de la encuesta aplicada se muestran en la Tabla 8, cuyas posibles respuestas son: totalmente de acuerdo (TA), de acuerdo (A), en desacuerdo (D) y totalmente en desacuerdo (TD). En la pregunta 1, la mayoría de los estudiantes no considera que el robot realiza mejores dictados que el profesor; en la segunda pregunta, la mayoría estuvo de acuerdo con la apariencia física del robot; en la pregunta 3, los estudiantes se dividieron, la mitad considera exitosa la tutoría presentada por el robot pero la otra estuvo en desacuerdo; en la pregunta 4, la mayoría de los estudiantes opinó que se deberían realizar actividades similares dentro de la asignatura utilizando el robot LRS1; finalmente, en la pregunta 5 , todos estuvieron totalmente de acuerdo en que el robot los motiva en el aprendizaje de la programación. En general, se refleja que la estrategia experimental presentada permite estimular y motivar el interés de los estudiantes dentro de la asignatura.

\section{Tabla 8. Resultados de la encuesta.}

\begin{tabular}{|c|c|c|c|c|c|}
\hline $\mathbf{N}$ & Preguntas & TA & $\mathbf{A}$ & D & TD \\
\hline 1 & $\begin{array}{l}\text { ¿Considera usted que el } \\
\text { robot realiza mejores } \\
\text { dictados que el profesor? }\end{array}$ & & 1 & 7 & 2 \\
\hline 2 & $\begin{array}{l}\text { ¿Considera usted que la } \\
\text { apariencia física del robot } \\
\text { es aceptable? }\end{array}$ & 3 & 7 & & \\
\hline 3 & $\begin{array}{l}\text { ¿Considera usted que la } \\
\text { tutoría del robot fue } \\
\text { exitosa? }\end{array}$ & & 5 & 5 & \\
\hline 4 & $\begin{array}{l}\text { ¿Considera usted que el } \\
\text { robot debería realizar otras } \\
\text { actividades dentro de la } \\
\text { asignatura? }\end{array}$ & 5 & 3 & 2 & \\
\hline 5 & $\begin{array}{l}\text { ¿Considera usted que el } \\
\text { robot puede motivar en el } \\
\text { aprendizaje de } \\
\text { programación? }\end{array}$ & 10 & & & \\
\hline
\end{tabular}

\section{ANÁLISIS}

El análisis se realiza en dos dimensiones: incidencia del robot social en los estudiantes de "Programación 1" y construcción de un robot social de bajo costo.

\subsection{Incidencia de LRS1 en los estudiantes}

Las calificaciones finales de los dictados favorecen al grupo de control porque su promedio es mayor, sin embargo, los resultados son relativamente cercanos. Los resultados parciales de cada dictado favorecen al grupo experimental porque obtuvo un mejor desempeño en los últimos dos dictados. De las observaciones realizadas durante el experimento, el mal desempeño del grupo experimental en el primer dictado, quizás está relacionado con la ubicación inicial de algunos estudiantes, porque cuando el robot comenzó a dictar, ellos se acercaron más al robot.

Los resultados de la encuesta aplicada a los estudiantes muestran cuatro aspectos 
fundamentales: primero, una tendencia que favorece al profesor en el dictado de los ejercicios (pregunta 1); segundo, una equidad sobre el éxito de la tutoría del robot (pregunta 3); tercero, una tendencia a que se realicen otras actividades con el robot (pregunta 4); y cuarto, una opinión favorecedora y contundente sobre la motivación que genera el robot (pregunta 5).

\subsection{LRS1: Un robot social de bajo costo}

En la Tabla 9 se presentan algunos robots sociales utilizados en el contexto de la educación con sus respectivos costos. Aunque otros robots tienen mayor potencial que LRS1, en general se observa una tendencia ha subutilizar tales características en experimentos realizados en escenarios educativos. En ese sentido, LRS1 es un robot social de bajo costo que permite incorporar al salón de clases algunas dinámicas comunes en robots sociales, tales como dictados.

Tabla 9. Costos de robots sociales

\begin{tabular}{|l|c|}
\hline \multicolumn{1}{|c|}{ Nombre } & Costo \\
\hline PEPPER & $\$ 19.600$ \\
\hline DARwIn-OP & $\$ 12.000$ \\
\hline NAO & $\$ 10.000$ \\
\hline Nao torso & $\$ 1.500$ \\
\hline TurtleBot-1 & $\$ 1.050$ \\
\hline Bioloid Expert Kit & $\$ 1.044$ \\
\hline Keepon & $\$ 280$ \\
\hline LRS1 & $\$ 41$ \\
\hline
\end{tabular}

\section{CONCLUSIONES}

El robot social LRS1 construido se basa en tres habilidades principales: hablar, expresar emociones faciales (triste, normal y feliz) y mover el cuello (arriba, abajo, izquierda, derecha y centro). La Interfaz de Programación de Aplicaciones proporcionada por la biblioteca lasdai-ula permite establecer fácilmente la comunicación entre un computador y LRS1 a través del puerto USB para controlar el estado del robot mediante 14 comandos muy intuitivos (expresarFeliz, comenzarHabla, moverDerecha, etc.).

Los resultados finales obtenidos en la actividad "dictado" muestran un leve mejor desempeño en el grupo donde el profesor realizó el dictado, sin embargo, los resultados parciales de los dictados 2 y 3 muestran mejor desempeño en el grupo donde el robot realizó el dictado, por lo tanto, aunque los estudiantes opinan que el profesor realiza mejor los dictados, se puede decir que la incorporación del robot social a la actividad "dictado" fue exitosa.
Los resultados obtenidos en la encuesta permiten decir contundentemente que el robot social LRS1 motiva el aprendizaje de los estudiantes de la asignatura "Programación 1".

El costo de construir el robot social LRS1 es muy bajo si se compara con otros robots que son utilizados en el contexto educativo. En ese sentido, aunque las habilidades de LRS1 son más limitadas, hay muchas actividades que se pueden implementar en aras de seguir motivando el aprendizaje de los estudiantes. Adicionalmente, a petición de los mismos estudiantes, los trabajos futuros están orientados a proporcionar prácticas de programación para que ellos mismos programen las actividades del robot.

\section{RECONOCIMIENTO}

Esta investigación fue parcialmente financiada por el CDCHTA-ULA a través del Proyecto número I1480-17-02-B.

\section{REFERENCIAS}

Blar N., Idris S., A., Jafar F., y Ali M. (2014). Robot and human teacher, International Conference on Computer, Information and Telecommunication Systems (CITS).

Brown L., y Howard A. (2014). The Positive Effects of Verbal Encouragement in Mathematics Education Using a Social Robot, IEEE Integrated STEM Education Conference.

Campbell D., y Stanley J. (1995). Diseños experimentales y cuasiexperimentales en la investigación social, edición en castellano por Amotrorlu editores, S. A., Paraguay 1225, $7 \mathrm{mo}$ piso, Buenos Aires.

Causo A., Win P., Guo P., y Chen I. (2017). Deploying Social Robots as Teaching Aid in Pre-school K2 Classes, IEEE International Conference on Robotics and Automation (ICRA).

Chin K., Hong Z., y Chen Y. (2014). Impact of Using an Educational Robot-Based Learning System on Students' Motivation in Elementary Education, IEEE Transactions on Learning Technologies.

Dapena E., Pérez J., Rivas R., y Guijarro A. (2016). Rostro genérico para máquinas 
que interactúan con personas. Revista científica UNET, 28(2):121-130.

Diprose J. (2014). Tools for programming human robot interaction, IEEE Symposium on Visual Languages and Human-Centric Computing (VL/HCC).

Diprose J., Plimer B., MacDonald B., y Hosking J. (2014). A Human-Centric API for Programming Socially Interactive Robots, IEEE Symposium on Visual Languages and Human-Centric Computing (VL/HCC).

Diyas Y., Brakk D., Aimambetov Y., y Sandygulova A. (2016). Evaluating peer versus teacher robot within educational scenario of programming learning, $11^{\text {th }}$ ACM/IEEE International Conference on Human-Robot Interaction (HRI).

El-Aawar H. (2017). Friendly re-programmed social robot, IEEE $26^{\text {th }}$ International Symposium on Industrial Electronics (ISIE).

Fu G., y Zhang X. (2015). ROSBOT: A low-cost autonomous social robot, IEEE International Conference on Advanced Intelligent Mechatronics (AIM).

Kahn Jr. P., Kanda T., Ishiguro H., Gill B., Shen S., Ruckert J., y Gary H. (2016). Human creativity can be facilitated through interacting with a social robot. In The Eleventh ACM/IEEE International Conference on Human Robot Interaction (pp. 173-180). IEEE Press.

Kennedy J., Baxter P., Senft E., y Belpaeme T. (2016). Social robot tutoring for child second language learning, $11^{\text {th }}$ ACM/IEEE International Conference on Human-Robot Interaction (HRI).

Kirstein F., y Risager R. (2016). Social robots in educational institutions they came to stay: Introducing, evaluating, and securing social robots in daily education, $11^{\text {th }}$ ACM/IEEE International Conference on Human-Robot Interaction (HRI).

Lemaignan S., Jacq A., Hood D., García F., Paiva A., y Dillenbourg P. (2016). Learning by Teaching a Robot: The Case of
Handwriting. IEEE Robotics \& Automation Magazine.

Meiirbekov S., Balkinberkov K., Jalankuzov Z., y Sandygulova A. (2016). "You win, I lose”: Towards adapting robot's teaching strategy, $11^{\text {th }}$ ACM/IEEE International Conference on Human-Robot Interaction (HRI).

Núñez P. (2014). Social robot as an assistive tool in the classroom, $9^{\text {th }}$ International Conference on Computer Science \& Education.

Pantecouteau, H., y Passera B. (2017), Brice. Influence of Human Personality Traits on Trust in Human-Robot Interactions. Master Recherche IC2A Ingénierie de la Cognition, de la Création et des Apprentissages, (pp. 62-69).

Sequeira P., Alves-Oliveira P., y Paiva A. (2016). The role that an educational robot plays, $25^{\text {th }}$ IEEE International Symposium on Robot and Human Interactive Communication (RO-MAN).

Sojib N., Islam S., Rupok M., Hasan S., Amin M., R., y Iqbal M. (2017). Design and development of the social humanoid robot named Ribo, IEEE Region 10 Humanitarian Technology Conference (R10-HTC).

Strohkorb S., Fukuto E., Warrren N., Taylor C., Berry B., y Scassellati B. (2016). Improving Human - Human collaboration between children with a social robot. In robot and Human Interactive Communication (RO-MAN), $201625^{\text {th }}$ IEEE international Symposium on (pp. 551-556). IEEE.

Tozadore D., Pinto A., Ranieri C., Batista M., y Romero R. (2017). Tablets and humanoid robots as engaging platforms for teaching languages, Latin American Robotics Symposium (LARS) and 2017 Brazilian Symposium on Robotics (SBR). 